Peculiar Versus Normal Phenomena in A-Type and Related Stars

ASP Conference Series, Vol. 44, 1993

M.M. Dworetsky, F. Castelli, and R. Faraggiana (eds.)

\title{
HYDROGEN-LINE PROFILES OF SOME $\lambda$ BOOTIS STARS
}

\author{
ILIAN KH. ILIEV \& INA S. BARZOVA \\ National Astronomical Observatory \\ p.o.box 136, Smolyan, BG - 4700, Bulgaria
}

\begin{abstract}
The observed hydrogen-line profiles of four $\lambda$ Boo type stars - HD 111786, HD 125162, HD 179791 and HD 192640 are presented. Existence of two separate groups based on the shape of these lines is confirmed. Radial velocity $\left(V_{r}=-5 \pm 3 \mathrm{~km} \mathrm{~s}^{-1}\right)$ and projected rotational velocity (vsini $=130 \pm 10 \mathrm{~km} \mathrm{~s}^{-1}$ ) for HD 111786 are measured.
\end{abstract}

\section{INTRODUCTION}

According to the definition of Hauck and Slettebak (1983) Lambda Bootis-type stars "are A-F stars with metallic lines which are too weak for their spectral types, when the latter are determined from the ratio of their K-line to the Balmer-line strengths. They are distinguished from weak-lined Population II stars by the fact they have normal space velocities and moderately large rotational velocities".

This definition has initiated the renewed interest in the $\lambda$ Bootis stars during the last decade. Hauck (1986) has pointed out that many of the objects in Abt's (1984) list of stars with weak $\mathrm{Mg}$ II $4481 \AA$ line also meet the requirements of the working definition. Baschek and Slettebak (1988) studied the chemical composition of $10 \lambda$ Bootis stars grounded on IUE spectra. The main result didn't vary essentially from the earlier study of Baschek and Searle (1969). Heavier elements like $\mathrm{Mg}, \mathrm{Fe}$ and $\mathrm{Ni}$ are underabundant of about 0.5 dex, while $\mathrm{C}, \mathrm{N}$ and $\mathrm{O}$ seem to be overabundant or normal. The underabundance of the metals were confirmed photometrically in the well-known $\Delta$ a system (Maitzen and Pavlovsky, 1989). Later Hiev et al. (1990) and Bohlender and Landstreet (1990) have shown that $\lambda$ Bootis stars are non-magnetic.

Gray (1988) improved the working definition by adding the extension: "The Lambda Bootis stars fall into two distinct classes, based on the appearance of their hydrogen lines. The first class (NHL) is characterized by normal (early A-dwarf) hydrogen-line profiles, the second (PHL) by peculiar hydrogen-line profiles with weak cores and broad but often shallow wings". However, Baschek and Slettebak (1988) mentioned that this separation is founded on subtle differences in line profiles and shonld be confirmed by further observations. This remark became a starting point of our attempts to answer the question whether these two different classes of hydrogen-line profiles really exist. 
In this paper we represent the results of our study on the $\mathrm{H} \gamma$ and $\mathrm{H} \delta$ line profiles of fonr $\lambda$ Bootis stars. According to Gray (1988) one of them (the $\lambda$ Boo itself) belongs to NHL group, the others (HD 111786 and $29 \mathrm{Cyg}$ ) have PHL profiles. The fourth star (HD 179791) is not included in Gray's list.

\section{OBSERVATIONS AND SPECTRA REDUCTION}

The observations were carried out at 2-m RCC telescope of Bulgarian National Astronomical Observatory. The spectrograms for each star were obtained in conde-focus on blue Kodak plates and have a dispersion of $9 \AA \mathrm{mm}^{-1}$ with a resolution of $0.18 \AA$. The plates were scanned with Joyce Loebl microdensitometer and then processed to "normalized intensity/heliocentric wavelengths" form on PC/AT by nsing the software package ReWiA (Borkowski, 1988).

During the data reduction a special attention to three stages has been paid: noise filtration, drawing the continunm and spectra coaddition. The noise filtration procedure followed closely the ideas of Tukey (1977). Multiple median and cubic spline smoothing procedures were used. The smoothing was succeed by the data restoring in order to eliminate possible loss of information in the bottom of the spectral lines.

In our mind, drawing the continunm is most important step in data reduction. The reason is that operating with broad and well developed hydrogen lines it is easy to produce as an artifact the observed PHL by placing the continuum too low especially above the central parts of the lines. To avoid this the continum was drawn across the whole available spectrum, covering the region $\lambda \lambda 3700-4700 \AA \AA$.

After the noise filtration and placing the continnum over the every spectrogram the obtained spectra were normalized and then co-added. Co-adding procedure is widely described by Hill and Adelman (1986) and its main goal is the increasing of $S / N$ ratio. The coadditions of the spectrograms have been completed for the HD 125162 (9 plates), the HD 111786 (7 plates), the HD 179791 (6 plates) and for the HD 192640 (12 plates). So, a brief description of the spectra reduction looks in such a way - filtering, restoring, drawing the continuum, normalizing and co-adding.

Table I contains identification of the observed stars, their photometric indices (b-y) (from Baschek and Slettebak, 1988), their projected rotational velocities (from Uesngi and Fuknda, 1982) and their hydrogen-line peculiarities (from Gray, 1988). Besides this the number of co-added spectrograms and reached $S / N$ ratio are also listed. In the case of coaddition $S / N$ can be easily determined as the ratio "mean value/standard deviation".

Table I Observed $\lambda$ Boo stars

\begin{tabular}{ccccccc}
\hline HD & HR/Name & (b-y) & vsini & C & n & S/N \\
\hline 111786 & 4881 & .161 & - & PHL & 7 & 55 \\
125162 & $\lambda$ Boo & .051 & 100 & NHL & 9 & 60 \\
179791 & 7288 & - & 185 & - & 6 & 50 \\
192640 & $29 \mathrm{Cyg}$ & .101 & 75 & PHL & 12 & 65 \\
\hline \hline
\end{tabular}




\section{RESULTS AND CONCLUDING REMARKS}

First of all, in order to increase the $S / N$ ratio once again both wings of every hydrogen line were averaged to right-side one. Then, the observed line profiles were compared with Kurucz's (1979) theoretical profiles.

HD 111786: This is the most interesting star in our sample. Peculiar character of its hydrogen-line profiles is clearly visible in Fig.1(a). Central part of both profiles (up to $10 \AA$ from the line center) matches well theoretical curves for $T$ eff $=7500 \mathrm{~K}, \log g=4.0$. The "wing part" of the observed profiles (starting at $30 \AA$ from the center) can be fitted with theoretical profiles for $T e f f=8000 \mathrm{~K}$, $\log g=4.0$. There is a transition part between central region and the "wing". The spectrum of HD 111786 looks a little strange for $\lambda$ Bootis star with too many lines of neutral metals. This is mainly due to star's lower temperature. The temperature obtained from central part fitting $-7500 \mathrm{~K}$ is in agreement with (b-y) of the star. We agree with Gray (1988) that in the "wing region" the hydrogen-line profiles for Teff $=8000 \mathrm{~K}, \log g=4.0$ doesn't differ considerably from the $T e f f=9500 \mathrm{~K}, \log g=3.5$ profiles. But in the case of HD 111786 higher temperature (and lower $\log g$ ) satisfies neither photometric data, nor the appearance of the spectram. In addition, we were able to determine the radial velocity of the HD 111786, which is $-5 \pm 3 \mathrm{~km} \mathrm{~s}^{-1}$ and the projected rotational velocity $130 \pm 10 \mathrm{~km} \mathrm{~s}^{-1}$. These values tally with Andersen and Nordström (1977) statement: "Fast rotation and low radial velocity".

HD 125162: Observed $\mathrm{H} \gamma$ profile for this star together with the theoretical one for Teff $=8800 \mathrm{~K}, \log g=4.0$ are shown in Fig.1(b). This temperature is confirmed by (b-y) indication for the star (see Table 3 from Baschek and Slettebak, 1988). The $\lambda$ Boo itself has a spectrum with substantial lack of metal lines. Wings of the hydrogen lines are very broad and especially red part of $\mathrm{Hy}$ can be traced through the spectrum up to $80 \AA$ from the line center. This is a perfect example of $\lambda$ Bootis type star with normal hydrogen lines.

HD 179791: This is the fastest rotating star in our list. Observed $\mathrm{H} \gamma$ profile is shown in Fig.1(c) along with the theoretical profile for Teff $=8300 \mathrm{~K}$, $\log g=4.0$. Nearly the same temperature for this star denotes its $(\mathrm{B}-\mathrm{V})=+0.09$ (from Abt, 1984). As it can be seen from Fig. 3 HD 179791 belongs to the group with normal hydrogen profiles. The Mg II $4481 \AA$ line is strong and/or probaly blended.

$H D$ 192640: The observed $H \delta$ and $H \gamma$ profiles are compared with the theoretical ones in Fig.1(d). The Teff $=8200 \mathrm{~K}, \log g=4.0$ profile matches well central part of both lines, while the "wing region" can be fitted with Teff $=8500 \mathrm{~K}$, $\log g=4.0$. The lower temperature $-8200 \mathrm{~K}$ is in better accordance with (b-y). The Ca II $3933 \AA$ line is strong.

Only by way of illustration the central depths of the most prominent featores in the spectra of the observed stars Ca II $3933 \AA$ and $\mathrm{Mg}$ II $4481 \AA$ lines are listed in Table II. 


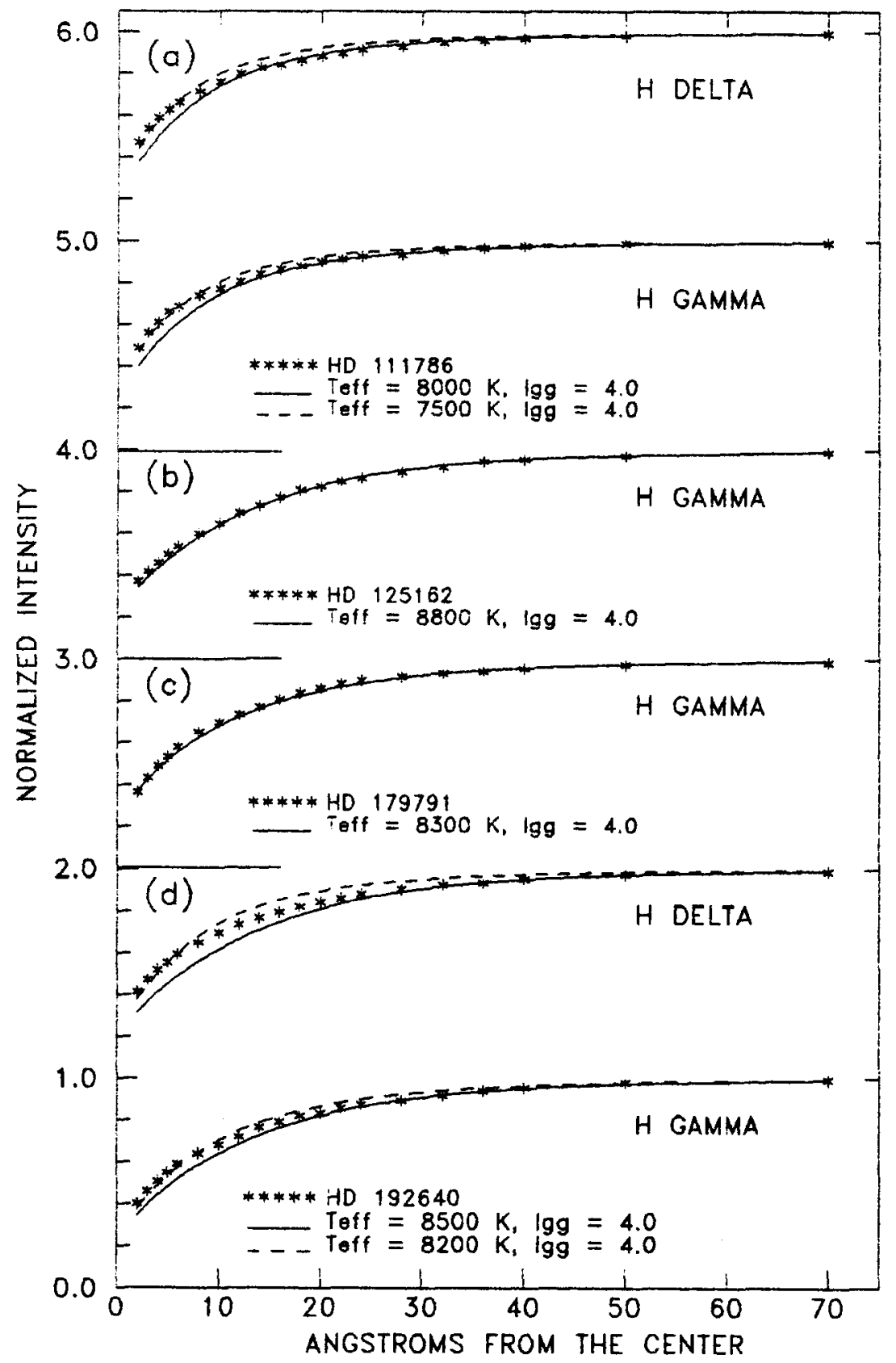

FIG. 1 - Observed and "best-fit theoretical" hydrogen-line profiles of HD 111786, HD 125162, HD 179791 and HD 192640. Note that top five groups of profiles are shifted. 
Table II Central depths

\begin{tabular}{ccc}
\hline HD & $3933 \AA$ & $4481 \AA$ \\
\hline 111786 & 0.59 & 0.94 \\
125162 & 0.82 & 0.95 \\
179791 & 0.50 & 0.87 \\
192640 & 0.44 & 0.91 \\
\hline \hline
\end{tabular}

Finally, it is worth noting that in the cases of peculiar hydrogen lines (HD 111786 and HD 192640) theoretical profiles for lower temperature fit the central part of the lines, whereas the "wing region" can be fitted with higher temperature profile. There is a good agreement between the temperatures of all observed stars derived from their photometric indices and the temperatnres obtained from their hydrogen lines. For both PHL stars the temperature obtained "photometrically" is closer to the temperature determined from those theoretical profiles that fit the central part of the hydrogen lines.

\section{REFERENCES}

Abt, H.A. 1984 in The $M K$ Process and $M K$ Classification, ed. R.F. Garrison, David Dunlop Observatory, p. 340.

Andersen, J., and Nordström, B. 1977, Astr. Ap. Suppl., 29, 309.

Baschek, B., and Searle, L. 1969, Ap. J., 155, 537.

Baschek, B., and Slettebak, A. 1988, Astr. Ap., 207, 112.

Bohlender, D.A., and Landstreet, J.D. 1990, M.N.R.A.S., 247, 606.

Borkowski, J. 1988, ReWiA-Instrukcija obslugy, (Poland: Tornn Observatory), (in polish).

Gray, R.O. 1988, A. J., 85, 220.

Hanck, B. 1986, Astr. Ap., 164, 349.

Hauck, B., and Slettebak, A. 1983, Astr. Ap., 127, 231.

Hill, G., and Adelman, S.J. 1986 in Upper Main Sequence Stars with Anomalous Abundances, eds. C.R.Cowley, M.M.Dworetsky and C.Megessier, (Dordrecht: Reidel), p. 209.

Diev, I.Kh., Barzova, I.S., Glagolevsky, Yn.V., Bychkov, V.D., Elkin, V.G., Romanjuk, I.I. and Shtohl, V.G. 1990, Mitteilnngen des K.Schwarzschild Obs. Tautenburg, Nr. 125, p. 78.

Kurucz, R.L. 1979, Ap. J. Suppl., 40, 1.

Maitzen, H.M., and Pavlovsky, K. 1989, Rev. Mexicana Astr. Astrof., 19, 54.

Tukey, J.W. 1977, Exploratory Data Analysis (Reading, MA: Addisson-Wesley Publishing Company).

Uesugi, A., and Fuknda, I. 1982, Revised Catalogue of Stellar Rotational Velocities (Kyoto: Kioto University). 


\section{DISCUSSION (liev and Barsova)}

CORBALLY: The unexplained absorption in the UV for some $\lambda$ Boo stars prompts me to invite you to speculate on the origin of the PHL (peculiar hydrogen line) feature that you have confirmed in one class of the $\lambda$ Boo stars.

ILIEV: In our mind there are at least two reasons for the observed PHL's. One of them is connected with abnormal structure of the stellar atmosphere, which can be produced by energy redistribution from the UV to the visible region, for example. The second cause may lie outside the star. Possibly there is cool gas surrounding it which could produce such a weak core as that we observe.

NORTH: In some young associations, some stars have abnormally broad hydrogen lines, and are classified as having luminosity class $\mathrm{Vb}$. Did you look for any link between these stars and $\lambda$ Boo stars?

ILIEV: No, we did not. But a common mechanism responsible for all these peculiarities ought to exist. This mechanism can be searched for; I am thinking now about the influence of circumstellar matter (gas and/or dust). In this connection, $\lambda$ Boo itself has an IR excess and normal hydrogen line profiles. On the other hand, HD 111786 has peculiar profiles and there is no evidence of circumstellar gas around it. 